\title{
Keratin 8 expression in colon cancer associates with low faecal butyrate levels
}

\author{
Abdul Q Khan ${ }^{1,2}$, Jonathan P Bury', Steven R Brown², Stuart A Riley², Bernard M Corfe ${ }^{1 *}$
}

\begin{abstract}
Background: Butyrate has been implicated in the mechanistic basis of the prevention of colorectal cancer by dietary fibre. Numerous in vitro studies have shown that butyrate regulates cell cycle and cell death. More recently we have shown that butyrate also regulates the integrity of the intermediate filament (IF) cytoskeleton in vitro. These and other data suggest a link between the role of diet and the implication of a central role for the keratin 8 (K8) as guardian of the colorectal epithelium.

Methods: In this cross-sectional study possible links between butyrate levels, field effects and keratin expression in cancer were addressed directly by analysing how levels of expression of the IF protein K8 in tumours, in adjacent fields and at a distant landmark site may be affected by the level of butyrate in the colon microenvironment. An immunohistochemical scoring protocol for K8 was developed and applied to samples, findings were further tested by immunoblotting.

Results: Levels of K8 in colorectal tumours are lower in subjects with higher levels of faecal butyrate. Immunoblotting supported this finding.Although there were no significant relationships with butyrate on the nontumour tissues, there was a consistent trend in all measures of extent or intensity of staining towards a reduction in expression with elevated butyrate, consistent with the inverse association in tumours.

Conclusions: The data suggest that butyrate may associate with down-regulation of the expression of K8 in the cancerized colon. If further validated these findings may suggest the chemopreventive value of butyrate is limited to early stage carcinogenesis as low K8 expression is associated with a poor prognosis.
\end{abstract}

\section{Background}

The cytoskeleton of epithelial cells includes three principal types of filaments: microfilaments, microtubules, and the intermediate filaments. Intermediate filaments are formed from heteropolymers of type I and type II keratins in epithelia, specifically keratin 8 (K8) and keratin 18 (K18) in the colon, with some expression of K7, K19, and K20. Dimerisation of keratins is mediated through pairing along their coiled-coil domains, and is regulated, at least in part, through post-translational modification of the globular domains outside these regions. Keratins provide ability to the epithelium to withstand mechanical strength as evident by the pathological phenotype seen in patients with mutations in epidermal keratins $[1,2]$. Analysis of the K8-null mouse revealed severe

\footnotetext{
* Correspondence: b.m.corfe@shef.ac.uk

'Department of Oncology, University of Sheffield, Medical School, Beech Hill Road, Sheffield, S10 2JF, UK

Full list of author information is available at the end of the article
}

disease of gastrointestinal tract characterized by colorectal hyperplasia and inflammation [3].

Keratins not only provide mechanical strength but are also involved in various regulatory functions of the cells. Genetic knock-out experiments have revealed distinct regulatory functions of $\mathrm{K} 8$ and $\mathrm{K} 18[4,5]$. K8 plays an important function in protecting the placental barrier function [6]. K8 and K18 may regulate cell cycle and cell growth: it is suggested that the absence of $\mathrm{K} 8$ or K18 disturbs the cell cycle, drives cells into the G2-S phase and leads to aberrant cytokinesis through phosphorylation of keratin and interaction with adaptor protein 14-3-3 [7]. It has been demonstrated that K8/K18 loss in mice leads to reduced hepatic size and protein synthesis [8]. K8/K18 provide resistance to apoptosis on stress and injury, and this effect may be mediated through their effect on the death receptors (DR), Fas and TNF- $\alpha$. K8/K18-null mouse hepatocytes were less resistant to Fas-mediated apoptosis [9]. Various

\section{() Biomed Central}


abnormalities have been described in $\mathrm{K} 8$ deficient mice including colonic hyperplasia $[10,11]$, hypersensitivity of the liver to stress $[3,12]$, and alterations in intestinal epithelial membrane proteins [13].

Human mutations in $\mathrm{K} 8$ or K18, the principal keratins of gastrointestinal tract, have been shown to be associated with cryptogenic cirrhosis and other liver disorders [14]. Pancreatitis has also been shown an association with $\mathrm{K} 8$ mutation [15]. A subset of patients with ulcerative colitis has been shown to carry missense mutations in the $\mathrm{K} 8$ or $\mathrm{K} 18$ genes. Reconstructions of these mutations in vitro (K8: G62C, $163 \mathrm{~V}, \mathrm{~K} 464 \mathrm{~N}$; K18: $\mathrm{S} 230 \mathrm{~T}$ ) resulted in reduced filament assembly [16]. A proteomic analysis of morphologically normal mucosa from three group of colorectal patients: cancer, polyp and normal showed the appearance of isoforms of $\mathrm{K} 8$ in apparently normal mucosa in polyp and cancer patients, compared with patients with no pathology [17]. Four K8 isoforms appeared in polyp mucosa relative to normal mucosa and seven $\mathrm{K} 8$ isoforms appeared in cancer mucosa relative to healthy mucosa. These findings indicate alterations in $\mathrm{K} 8$, either at the level of expression or modification in the morphologically normal mucosa as the adenoma-carcinoma sequence progresses. Another study showing differential K8 expression in colorectal carcinoma has shown reduced expression of K8 in colorectal cancer is significantly associated with shorter patient's survival, possibly on the basis of epithelial-mesenchymal transition [18]. Taken together the data from studies of both ulcerative colitis and adenocarcinoma pathologies suggests an important role for K8 and $\mathrm{K} 18$ in maintenance and stability of intestinal epithelia. A characteristic of keratins is their relative stability of expression even after transformation to pathological state including transformation of normal cells into malignant cells. This property has enabled keratins to be applied as tumour markers [19].

Many epidemiological studies show an inverse relationship between dietary fibre intake and the incidence of colorectal cancer, including the large EPIC study [20]. One potential mechanism for the chemoprotective effect of fibre is through the production of short-chain fatty acids (SCFA) by fermentation in the colon lumen and regulation of epithelial homeostasis. Among the SCFAs butyrate is considered to be responsible for this chemoprotection as it has major influence on cell cycle, cell differentiation and cell death. Butyrate has been shown to induce growth inhibition and terminal differentiation in a variety of human colon cancer cell lines [21-23]. Butyrate also triggers apoptosis in various cell lines [24]. The ability of butyrate to alter various cell functions is considered to be through ability to regulate gene expression by inhibition of histone deacetylase (HDAC) $[25,26]$. This results in hyperacetylation of histone and enhancement of transcription factors and activation or modulation of acetylated transcription factors such as Sp1 [27], nuclear structural proteins [28], and p53 [29].

Given the importance of keratin expression and function to gastrointestinal function as evidenced by the knockout mouse and heritable predisposition studies, coupled with the ability of the chemopreventive butyrate to regulate expression and function of proteins, we sought to investigate what the effect of butyrate levels in the colon microenvironment have upon expression and localisation of $\mathrm{K} 8$ in the normal and neoplastic colon.

\section{Methods}

\section{Recruitment and SCFA extraction}

A total of 17 patients with colorectal cancer were recruited for this study, 4 patients from surgical lists and 13 from endoscopy lists [30]. Biopsies were taken from three different sites in each patient as shown in Table 1. All biopsies taken for immunohistochemistry (IHC) were fixed in formalin and embedded in paraffin. Stool samples were collected from each patient at least two weeks after endoscopic procedures, for patients recruited from Endoscopy list. Stool samples for patients recruited from surgical lists were collected before surgery. Stools were processed for SCFA extraction and butyrate concentrations determined by gas chromatography [31]. Ethics committee approval was obtained from the North Sheffield Research Ethics Committee prior to recruiting (Reference number: 06/Q2308/93). Subjects included in this study were recruited between October 2007 and June 2008.

\section{Immunohistochemistry}

All biopsies were fixed for 24 hours in formalin before paraffin embedding and cutting of serial 4-micron sections at 40-micron intervals. Antigen retrieval was performed with EDTA (1 mM, pH8) in microwave at high

\section{Table 1 Biopsying strategy for this study}

\begin{tabular}{|c|c|c|}
\hline Diagnosis & Biopsy positions & Other samples \\
\hline \multirow[t]{7}{*}{ Cancer } & $\begin{array}{l}2 \times \text { Mid-sigmoid for } \\
\text { proteomics }\end{array}$ & $\begin{array}{l}\text { Stool, for short-chain fatty acid } \\
\text { determination, including butyrate }\end{array}$ \\
\hline & $\begin{array}{l}1 \times \text { mid-sigmoid for } \\
\mathrm{IHC}\end{array}$ & \\
\hline & $\begin{array}{l}1 \times \text { mid-sigmoid for } \\
\text { whole mount }\end{array}$ & Food frequency questionnaire \\
\hline & $\begin{array}{l}2 x \text { contralateral wall } \\
\text { (field) for proteomics }\end{array}$ & \\
\hline & $\begin{array}{l}1 \times \text { contralateral wall } \\
\text { (field) for } \mathrm{HCC}\end{array}$ & \\
\hline & $\begin{array}{l}2 \times \text { lesion for } \\
\text { proteomics }\end{array}$ & \\
\hline & 1x lesion for IHC & \\
\hline
\end{tabular}


power for 8 mints. Avidin-biotin immunoperoxidase technique was used and diaminobenzidine was used as chromogen. Vectastain Universal RTU elite ABC kit, pk7200 was used for blocking agent, biotinylated secondary and $\mathrm{ABC}$ reagent.

Sections were blocked with normal horse serum for 30 minutes at room temperature and then incubated with primary antibody (K8 mouse monoclonal M20 [32] from Abcam ab9023,, 1:200 dilution) for 60 minutes at room temperature. Sections were incubated then with Biotinylated secondary antibody (anti mouse IgG from RTU Vectastain Universal elite ABC kit, Pk-7200) for 30 minutes followed by incubation with $\mathrm{ABC}$ reagent for 30 minutes. PBS (phosphate buffered saline) was used for washes in between. Detection was performed using DAB kit (Vector lab- sk-4100).

\section{IHC Scoring for K8}

Two slides containing three sections of each biopsy were stained. A maximum of 6 well-oriented crypts per biopsy, showing entire length of the crypt wall from the base abutting the muscularis mucosa through to the junction with the surface epithelium, were scored. Images were captured at $20 x$ magnification with a Nikon D5-M camera at $2560 \times 1920$ resolution, stored without compression and analysed using Nikon NIS-Elements D (v 2.30) software. Scoring was performed by one observer blind to the status of the biopsy. A subset of observations was confirmed by a second independent observer.

\section{Protein extraction and immunoblotting for K8}

Eight pinch biopsies were selected according to butyrate levels (4 high and 4 low). A modification of the intermediate filament extraction protocol described by Herman et al [33] was performed, specifically using the insoluble residue from biopsy lysis. The high-salt insoluble fraction (containing enriched intermediate filament proteins) was boiled in Laemmli buffer and separated by SDS-Page prior to immunoblotting. Primary antibody (K8 mouse monoclonal, ab9023) was used at 1:1000 concentration. The cross-reaction was visualized using HRP-conjugated secondary antibodies (Dako mouse for K8) and Western Lightning Chemiluminescence reagent plus (PerkinElmer, Boston, USA). Imaging was performed with Chemigenius Bio-Imaging system (Syngene).

\section{Statistical tests}

Graph Pad Prism 5.02 was used for statistical analysis. Unpaired t-test was used to compare mean scores of K8 expression, including mucosal surface intensity, crypt staining intensity and crypt staining extent between mid sigmoid (MS) and contra lateral wall (CO). Unpaired t-test was also used to find relationship of two groups of butyrate with $\mathrm{K} 8$ expression in different parts including cancer sites. Relationship of faecal butyrate levels and K8 expression for all sites was also studied using Pearson correlation.

\section{Results}

\section{Development of scoring criteria}

Each biopsy from contralateral wall (CO) and mid sigmoid (MS) area was scored in three categories: surface staining intensity, crypt staining intensity relative to surface staining, and extent of crypt staining. A maximum of six crypts from six available sections on two slides were chosen for scoring from each biopsy. Means were calculated for each category scored. Intensity of surface staining was scored from 3 to 0 , where 3 represented the best staining and 0 being minimal or no staining. Intensity of crypt staining was compared with intensity of surface staining and was scored 3 if better than surface staining, 2 if similar to surface staining and 1 if less than surface. Zero score was awarded if minimal or no staining observed in crypt. Score 3 was awarded if the whole extent of the crypt was stained. Score 2 being $2 / 3$ and 1 being $1 / 3$ of the crypt. If only few random cells were stained, that section was considered negative for scoring purposes. Immunohistochemical sections from cancer sites were scored from 0 to 3 on the basis of proportion of cells showing positive staining. Score 3 if more than $60 \%$ of cells were positively stained for K8; 2 if around half of the cells stained and 1 if less than $40 \%$ of the cells were stained with $\mathrm{K} 8$.

\section{Variation in K8 staining in normal tissue}

K8 staining was observed as diffusely distributed in the cytoplasm of the epithelial cells (Figure 1A). Expression of K8 was noted to varying degrees along the crypt villus axis, and with varying intensities in the crypt and at the mucosal surface. In total, immunohistochemical data was available for 17 patients. Fifteen patients had good sections for scoring purposes from both (MS and $\mathrm{CO})$ sites.

K8 mucosal surface staining intensity was not uniform in all sections. Examples of various staining intensities at mucosal surface are shown in Figure 1A. Figure 1, Panel Ai shows a weak staining intensity of $\mathrm{K} 8$ at the surface mucosa (score1) compared to Panel Aii (score2) and Panel Aiii which shows the highest staining intensity (score3). Although there was variation of staining intensity among biopsies from different patient at both sites, there was consistency among the sections from the same biopsy. When staining intensities of crypts (at surface mucosa) were compared between the landmark and the contralateral sites, $52 \%$ 


\section{A}
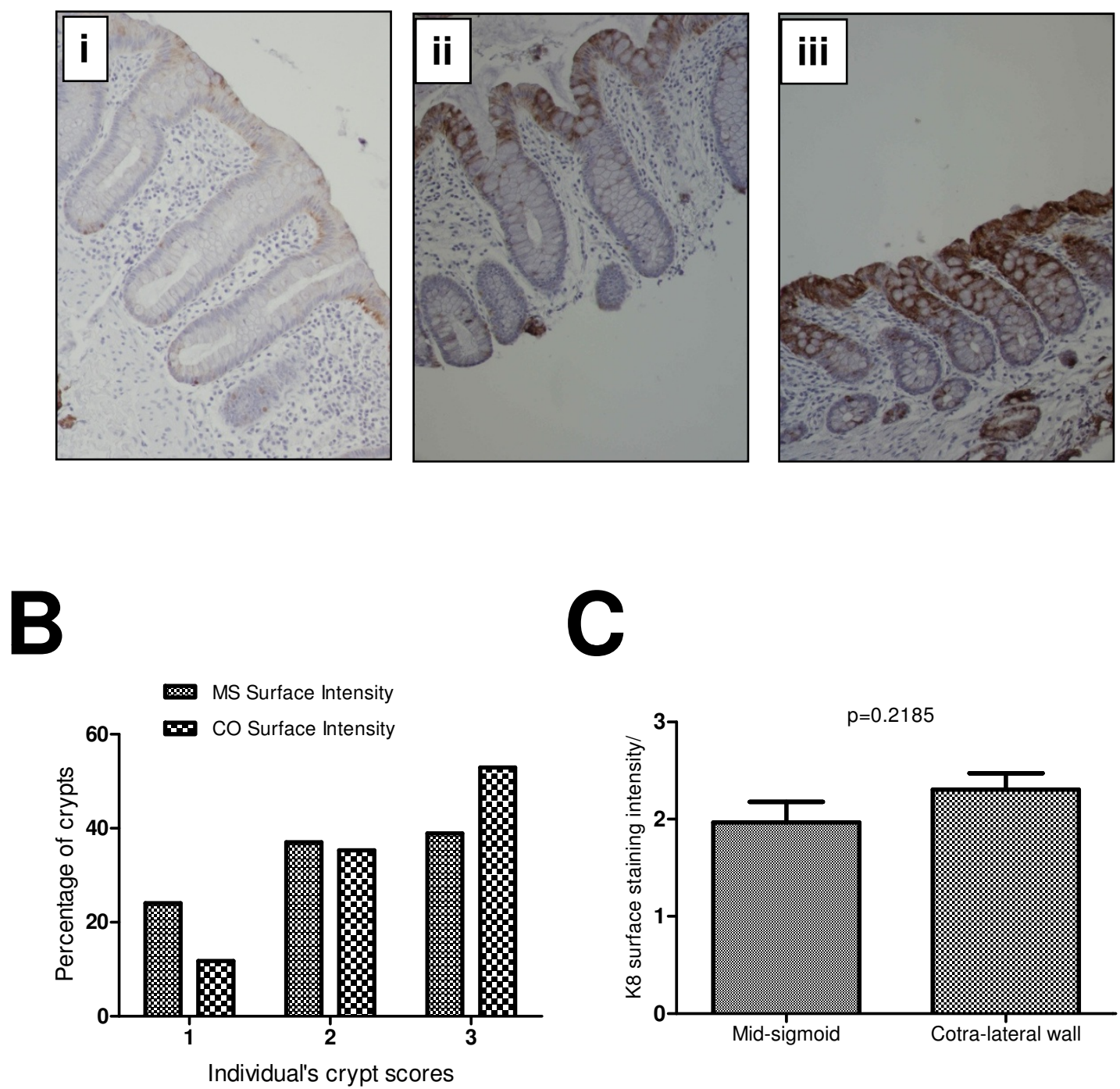

Figure 1 K8 surface expression at mid-sigmoid (MS) and contra-lateral wall (CO). (A) Examples of various surface staining intensities. A weak staining at surface mucosa was scored 1 (Panel 1Ai) and the strong intensity was scored as 2 (Panel 1Aii). (B) Most common pattern both at MS and CO was 3 suggesting that K8 expression is most strongly expressed at the mucosal surface (38.88\% at MS and 52.94\% at CO). (C) Unpaired t-test shows a high mean value (2.3) at CO compared with MS (1.96) indicating a trend towards a higher surface expression of K8 around cancer compared to the distant mucosa.

crypts shows strong staining intensity compared to $38 \%$ at the landmark site, with correspondingly fewer sites showing weak staining (Figure 1B). These data indicate a trend towards an increase in staining intensity at surface mucosa during progression towards the cancer from normal mucosa of the mid-sigmoid. This is also evident from higher mean score $(2.3)$ at $\mathrm{CO}$ compared to MS (1.96) score (Figure 1C).

Examples of differing degrees of staining along the crypt-villus axis are shown in Figure 2A. Figure 2A.i shows an example of staining reaching the bottom third of the crypt, whereas Figure 2A.iii shows an example of K8 expression with poor crypt penetrance. Intermediate examples, as shown in Figure 2A.ii, were also observed. There was general within-subject consistency in terms of staining pattern. The relative occurrence of each class of staining extent is shown in Figure 2B. Most common pattern seen both at MS (46.24\%) and CO (53.57\%) was 3 , showing that staining reached up to lower third of the crypt axis. In contrast to the findings for staining 

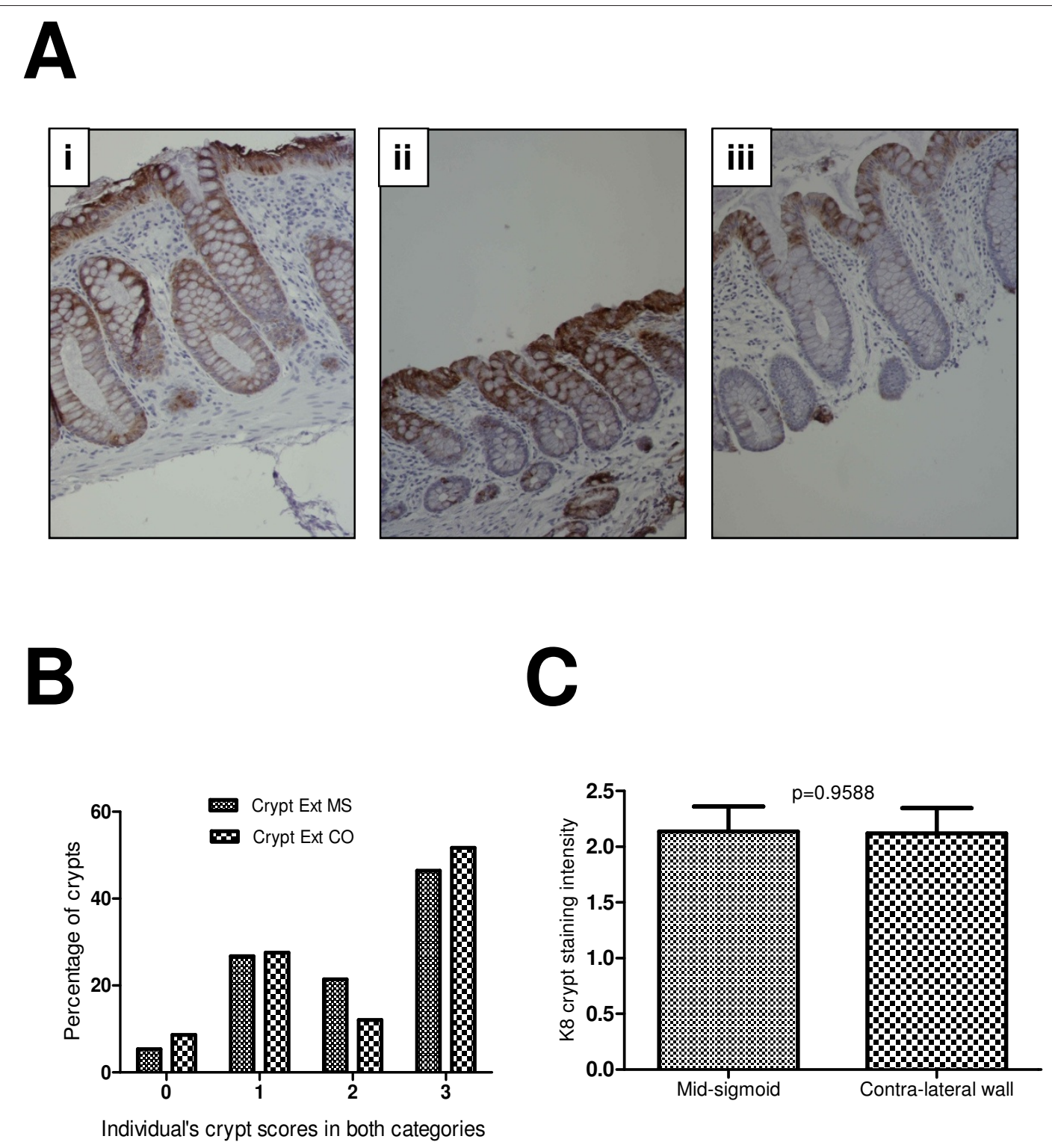

Figure 2 K8 expression along the crypt axis. Figure 2Ai shows K8 staining reaching to the bottom of the crypt, but in some crypts it reached only the upper one third (Aii) and in others upper two third was stained with K8 (A-iii). The relative occurrence of each class of staining is show in Figure 2B. Only few percent of crypts were without staining (5.37\% in MS group and $8.62 \%$ in CO group) but majority of the crypts scored K8 staining reached to the bottom in both MS and CO $(51.72 \%$ in CO and $46.24 \%$ in MS). There was no significant difference in the mean scores in two groups (C).

intensity, there was not a marked difference in the staining extent patterns between landmark and contralateral sites. A mean score of less than $2(1.75$ at MS and 1.66 at $\mathrm{CO}$ ) was observed for intensity of the crypt staining (Table 2) implicating the stronger mucosal staining compared to the intensity of crypt staining.

\section{K8 patterns and scoring in cancer tissue}

Biopsies from 14 patients' cancer tissues were stained for K8. There was a wide variation in staining patterns probably because of regional heterogeneity of the tissue. Only two samples showed a score of a less than 2 i.e. most cancer tissues showed at least 50\% expression of K8. None of the sample was negative for $\mathrm{K} 8$ which is consistent with previous results [34]. It was noted that highly undifferentiated tumours have a very patchy distribution of $\mathrm{K} 8$ (Figure 3-Aii) whereas more differentiated tumours have a pattern where cells along the mucosal surface were more positive for $\mathrm{K} 8$ and deep cells were either negative or showed less K8 expression (Figure 3A-iii). 
Table 2 Summary table of differences in staining between mucosal sites

\begin{tabular}{lccc}
\hline & Mid-sigmoid (mean) & Contralateral Wall (mean) & P value \\
\hline Surface staining intensity & 1.96 & 2.31 & 0.219 \\
Crypt staining intensity & 1.75 & 1.66 & 0.614 \\
\hline Extent of crypt staining & 2.13 & 2.12 & 0.959 \\
\hline
\end{tabular}
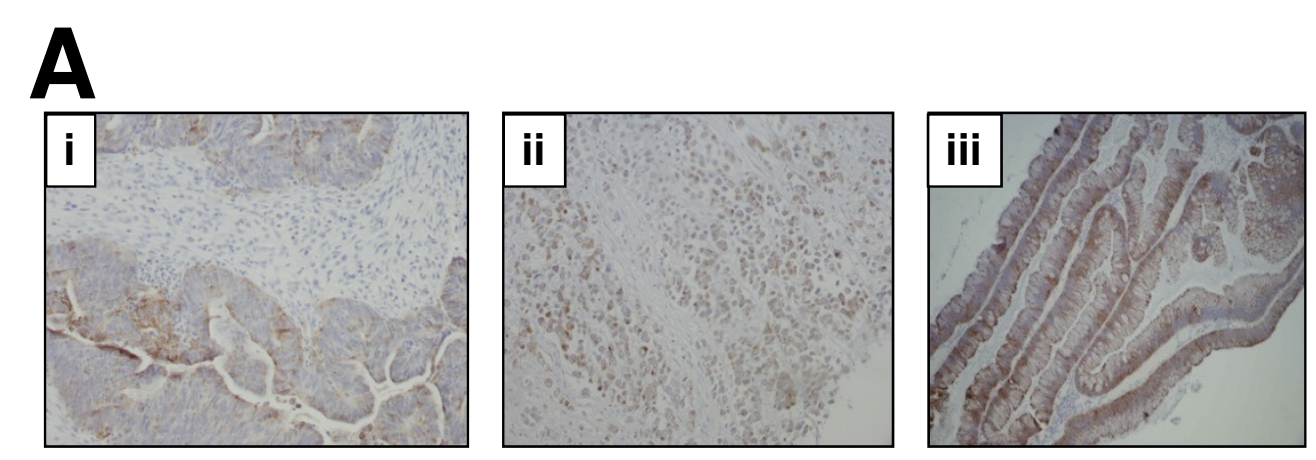

B
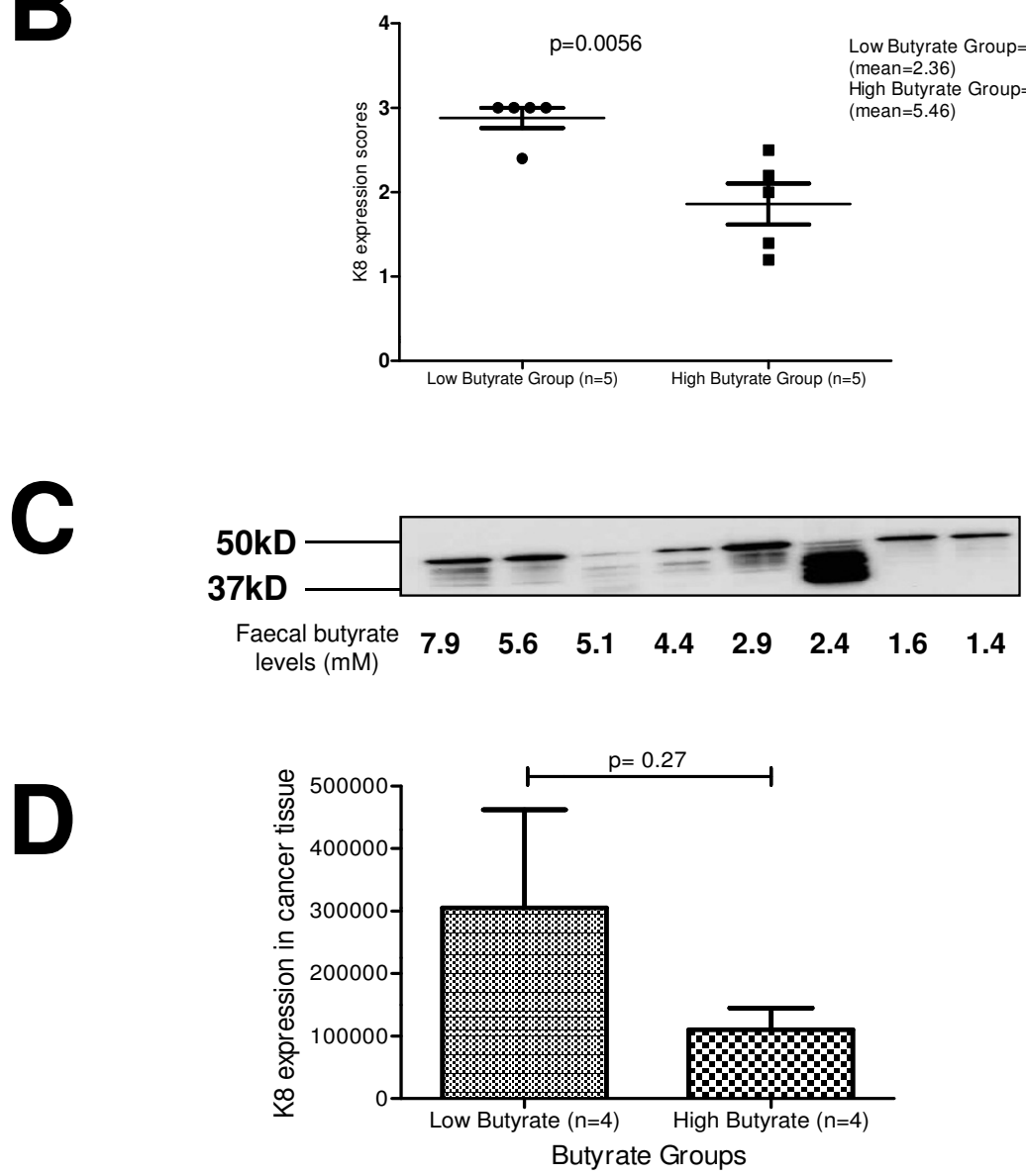

Figure $\mathbf{3}$ K8 expression in cancer tissue. Panels Ai-iii shows various examples of K8 expression scores in cancer tissue. Panel B: A significant difference of $\mathrm{K} 8$ expression in cancer tissue was observed between high (mean $=5.46 \mathrm{mM})$ and low butyrate $(\mathrm{mean}=2.36 \mathrm{mM}) \mathrm{groups}(\mathrm{p}=$ 0.0056). Panel $\mathrm{C}$ shows immunoblots for K8 in cancer tissue: a total 8 samples were used, 4 with high faecal butyrate levels and 4 with low faecal butyrate levels. Panel D: Densitometry result shows an inverse relationship between faecal butyrate levels and K8 expression in the cancer tissue. 
Relationship between faecal butyrate levels and K8 expression in morphologically normal mucosa

Faecal butyrate scores were available for 9 patients. Patients were split into haptiles by butyrate level (Low butyrate group: 0-4 mM, mean 2.36; High butyrate group $>4 \mathrm{mM}$, mean 5.54 ). At the morphologically normal sites there was no statistically significant difference in K8 expression between two butyrate groups in any of the three categories of scoring (Table 3) both at either mid sigmoid and field. However it was noted that all scores showed a decrease from low to high butyrate, indicating a consistent pattern or trend towards decreased expression of $\mathrm{K} 8$ with elevated butyrate. Pearson correlation coefficient (Table 4) also did not reveal any significant relationship between faecal butyrate levels and $\mathrm{K} 8$ expression in any scored categories at all three sites.

\section{High butyrate levels are associated with low K8 expression in cancer tissue}

Patient data was split into haptiles for butyrate level as described above. Low and high butyrate groups were compared for $\mathrm{K} 8$ expression (Figure $3 \mathrm{~B}$ ). A highly significant difference $(\mathrm{p}=0.0056)$ was observed in two groups with higher butyrate levels associated with a significant decrease in K8 staining. Intermediate filament proteins were extracted from duplicate samples and analysed for level of expression by immunoblotting. All cross-reacting bands were quantified for the scoring purposes (Figure 3C). Cross-reactions were quantitated from subjects' samples for higher and lower haptile by butyrate concentration. These results also suggested a similar inverse relationship between faecal butyrate levels and K8 expression in cancer tissue (Figure 3C \&3D) although the considerable variation between samples resulted in a non-significant finding.

\section{Discussion}

There is much emphasis on early detection of colorectal cancer and for that reason knowledge of various protein alterations which occur during carcinogenesis is important. K8 is one of major keratins in the colorectal mucosa. Through our scoring system we tried to investigate any progressive change in $\mathrm{K} 8$ expression from distant mucosa (MS in our study) to the cancer's field $(\mathrm{CO})$ to the cancer. There was no significant difference in crypt staining intensity and crypt staining extent but there was a clear trend towards higher K8 expression in the cancer's field when compared to the distant mucosa (MS) (Figure 1C). Polley et al. [17], using a proteomic approach, also showed that there is an increase in expression of some $\mathrm{K} 8$ isoforms in peri-adenomatous fields. Polley et al. were unable to identify the isoform, but there is consilience insofar as both independent studies find an increase in keratin 8 with cancer progression, including in the fields around cancer.

Our scoring system for K8 expression was used to study possible relationships between the faecal butyrate (as a proxy measure of lumenal butyrate), keratin expression and colorectal cancer. We hypothesized that K8 may be altered in colonic mucosa in response to butyrate. Development of a scoring system in three categories including mucosal surface staining intensity, crypt staining intensity, and extent of crypt staining allowed testing of this hypothesis. Expression of K8 in tumours was associated with butyrate level. These data were supported by western blot analysis using a K8-specific antibody to immunoprobe the insoluble protein (K8-rich) fraction after biopsy lysis. We noted the appearance of faster migrating forms on this blot. This could be attributed to an altered profile of phosphorylation, which has been shown to link with cancer progression in the colon [35], although phosphorylation at S73 and S431 was not associated with an alteration in protein mobility in gels, $\mathrm{K} 8$ is a highly modified molecule. It is our working hypothesis that these bands represent proteolytically cleaved forms associated with the cancer degradome [36].

Taken alone the reduction in keratin 8 expression associated with butyrate would be seen as a risk factor for tumour progression [18], although data would need to be interpreted against a wider panel protein alterations to establish whether the global risk was actually altered. A reduction in keratin expression is observed during epithelial to mesenchymal transition, where epithelial cytoskeletal proteins are replaced with proteins

Table 3 Summary table of differences in staining at mucosal sites by differing butyrate level

\begin{tabular}{|c|c|c|c|}
\hline \multirow[t]{2}{*}{ Tissue type } & \multicolumn{2}{|l|}{ Keratin expression score (mean) } & \multirow[t]{2}{*}{$P$ value } \\
\hline & Low butyrate $(\mathrm{n}=5$, mean $=2.4 \mathrm{mM}$ ) & High butyrate group $(\mathrm{n}=4$, mean $=5.5 \mathrm{mM}$ ) & \\
\hline MS surface intensity & 2.12 & 1.4 & 0.12 \\
\hline MS crypt intensity & 1.84 & 1.66 & 0.6 \\
\hline MS crypt extent & 2.24 & 1.76 & 0.44 \\
\hline CO surface intensity & 2.44 & 2.15 & 0.58 \\
\hline CO crypt intensity & 1.82 & 1.67 & 0.59 \\
\hline CO crypt extent & 2.12 & 2.0 & 0.85 \\
\hline
\end{tabular}


Table 4 Summary table of Pearson correlation coefficient between faecal butyrate levels and $\mathrm{K} 8$ expression at midsigmoid, contralateral wall, and carcinoma

\begin{tabular}{llll}
\hline & N & Correlation coefficient & P value \\
\hline Surface staining at MS & 9 & -0.209 & 0.59 \\
Surfance staining at CO & 9 & -0.087 & 0.82 \\
Crypt intensity staining at MS & 9 & -0.069 & 0.86 \\
Crypt intensity staining at CO & 9 & -0.145 & 0.71 \\
Extent of crypt staining at MS & 9 & -0.050 & 0.89 \\
Extent of crypt staining at CO & 9 & 0.014 & 0.97 \\
\hline Staining in cancer tissue & 10 & 0.050 & 0.15
\end{tabular}

Pearson correlation coefficient between faecal butyrate levels and K8 expression at mid-sigmoid, contralateral wall and carcinoma

more commonly associated with mesenchymal cells such as vimentin. Although not particularly widely described in colorectal oncology, progression through EMT is associated with a poor prognosis. There are precedents for nutrients associated with chemoprotective effects actually to elevate risk in the cancerized colon, notably folate [37].

One possible limitation of the study is the use of a single sampling point for faecal butyrate. A recent report highlighted the levels of intraindividual variation in SCFA concentration and SCFA production are labile, though it did suggest that concentrations were more stable as a measure than total output [38]. We reason that cells in the epithelium are exposed to a concentration, not a production rate, and have used this measure. We have found that other markers strongly correlate with faecal butyrate [39] suggesting relationships can be found, and that by implication the relationship between faecal butyrate and keratin expression is, at best, weak. A further hypothesis which may account for these findings could be that butyrate is a proxy measure of an unidentified bioactive secondary metabolite which is modulating keratin expression, or that direct interaction between butyrateproducing endosymbionts and the mucosa mediates this effect.

There was a consistent trend of increase in K8 expression in the morphologically normal tissues that did not reach significance. This observation merits further study to distinguish any possible causal role for $\mathrm{K} 8$ in cancer progression and the potential of manipulation of the colon luminal environment as a chemopreventive strategy.

\section{Conclusions}

The pattern of $\mathrm{K} 8$ expression in normal and cancer mucosa was reported by Fujiski [34]. Our data are consistent with their findings and extend their findings by providing a scoring system, by analysing the potential relationship between $\mathrm{K} 8$ staining and butyrate, and by cross-validating the data with a second independent methodology. Our data suggest there may be an increase in K8 expression from landmark to contralateral fields (supporting the findings of Polley et al [17]). We also suggest that there may be a very weak trend for butyrate to reduce expression of $\mathrm{K} 8$ in morphologically normal tissue, but that this relationship is stronger in tumour tissue.

Further studies could compare K8 expression in apparently normal mucosa from normal screened population and cancer patients, to establish whether there is any progressive and identifiable change in $\mathrm{K} 8$ expression during carcinogenesis.

\section{Acknowledgements}

This work was funded by Food Standards Agency (Ref: N12017) and BMI Thornbury Hospital Sheffield. We thank Prof Chris Seale \& Wendy Bal (Newcastle University) for GC analysis of faecal extracts.

\section{Author details}

'Department of Oncology, University of Sheffield, Medical School, Beech Hill Road, Sheffield, S10 2JF, UK. ²Department of Gastroenterology, Northern General Hospital, Herries Road, Sheffield, S5 7AU, UK.

\section{Authors' contributions}

AQK developed the staining method, recruited subjects, undertook all staining, scoring, western blotting and wrote the first draft of the paper; JPB supervised the development of staining and scoring protocols, undertook second scoring and supervised statistical analysis; SRB supervised and undertook surgical sampling, co-supervised the project with BMC and edited the draft manuscripts; SAR took overall clinical responsibility for the overarching study, undertook endoscopic sampling and edited the draft manuscripts; BMC conceived and directed the study and produced the final version of the manuscript. All authors read an approved the manuscript.

\section{Competing interests}

The authors declare that they have no competing interests.

Received: 18 May 2010 Accepted: 10 January 2011

Published: 10 January 2011

\section{References}

1. Coulombe PA, Hutton ME, Letai A, Hebert A, Paller AS, Fuchs E: Point mutations in human keratin 14 genes of epidermolysis bullosa simplex patients: genetic and functional analyses. Cell 1991, 66(6):1301-11.

2. Lane EB, Rugg EL, Navsaria H, Leigh IM, Heagerty AH, Ishida-Yamamoto A, et al: A mutation in the conserved helix termination peptide of keratin 5 in hereditary skin blistering. Nature 1992, 356(6366):244-6.

3. Loranger A, Duclos S, Grenier A, Price J, Wilson-Heiner M, Baribault H, et al: Simple epithelium keratins are required for maintenance of hepatocyte integrity. Am J Pathol 1997, 151(6):1673-83.

4. Magin TM, Vijayaraj $P$, Leube RE: Structural and regulatory functions of keratins. Exp Cell Res 2007, 313(10):2021-32.

5. Oshima RG: Intermediate filaments: a historical perspective. Exp Cell Res 2007, 313(10):1981-94.

6. Jaquemar D, Kupriyanov S, Wankell M, Avis J, Benirschke K, Baribault H, et al: Keratin 8 protection of placental barrier function. J Cell Biol 2003, 161(4):749-56.

7. Toivola DM, Nieminen Ml, Hesse M, He T, Baribault H, Magin TM, et al: Disturbances in hepatic cell-cycle regulation in mice with assemblydeficient keratins 8/18. Hepatology 2001, 34(6):1174-83.

8. Galarneau L, Loranger A, Gilbert S, Marceau N: Keratins modulate hepatic cell adhesion, size and G1/S transition. Exp Cell Res 2007, 313(1):179-94. 
9. Gilbert S, Loranger A, Daigle N, Marceau N: Simple epithelium keratins 8 and 18 provide resistance to Fas-mediated apoptosis. The protection occurs through a receptor-targeting modulation. J Cell Biol 2001, 154(4):763-73.

10. Baribault H, Penner J, lozzo RV, Wilson-Heiner M: Colorectal hyperplasia and inflammation in keratin 8-deficient FVB/N mice. Genes Dev 1994, 8(24):2964-73.

11. Habtezion A, Toivola DM, Butcher EC, Omary MB: Keratin-8-deficient mice develop chronic spontaneous Th2 colitis amenable to antibiotic treatment. J Cell Sci 2005, 118(Pt 9):1971-80.

12. Ku NO, Omary MB: A disease- and phosphorylation-related nonmechanical function for keratin 8. J Cell Biol 2006, 174(1):115-25.

13. Ameen NA, Figueroa $Y$, Salas PJ: Anomalous apical plasma membrane phenotype in CK8-deficient mice indicates a novel role for intermediate filaments in the polarization of simple epithelia. J Cell Sci 2001, 114(Pt 3):563-75.

14. Ku NO, Gish R, Wright TL, Omary MB: Keratin 8 mutations in patients with cryptogenic liver disease. N Engl J Med 2001, 344(21):1580-7.

15. Cavestro GM, Frulloni L, Nouvenne A, Neri TM, Calore B, Ferri B, et al: Association of keratin 8 gene mutation with chronic pancreatitis. Dig Liver Dis 2003, 35(6):416-20

16. Owens DW, Wilson NJ, Hill AJ, Rugg EL, Porter RM, Hutcheson AM, et al: Human keratin 8 mutations that disturb filament assembly observed in inflammatory bowel disease patients. J Cell Sci 2004, 117(Pt 10):1989-99.

17. Polley AC, Mulholland F, Pin C, Williams EA, Bradburn DM, Mills SJ, et al: Proteomic analysis reveals field-wide changes in protein expression in the morphologically normal mucosa of patients with colorectal neoplasia. Cancer Res 2006, 66(13):6553-62.

18. Knosel T, Emde V, Schluns K, Schlag PM, Dietel M, Petersen I: Cytokeratin profiles identify diagnostic signatures in colorectal cancer using multiplex analysis of tissue microarrays. Cell Oncol 2006, 28(4):167-75.

19. Chu PG, Weiss LM: Keratin expression in human tissues and neoplasms. Histopathology 2002, 40(5):403-39.

20. Bingham SA, Day NE, Luben R, Ferrari P, Slimani N, Norat T, et al: Dietary fibre in food and protection against colorectal cancer in the European Prospective Investigation into Cancer and Nutrition (EPIC): an observational study. Lancet 2003, 361(9368):1496-501.

21. Whitehead $\mathrm{RH}$, Young GP, Bhathal PS: Effects of short chain fatty acids on a new human colon carcinoma cell line (LIM1215). Gut 1986, 27(12):1457-63.

22. Comalada M, Bailon E, de Haro O, Lara-Villoslada F, Xaus J, Zarzuelo A, et al: The effects of short-chain fatty acids on colon epithelial proliferation and survival depend on the cellular phenotype. J Cancer Res Clin Oncol 2006, 132(8):487-97.

23. Augeron C, Laboisse CL: Emergence of permanently differentiated cell clones in a human colonic cancer cell line in culture after treatment with sodium butyrate. Cancer Res 1984, 44(9):3961-9.

24. Hague A, Elder DJ, Hicks DJ, Paraskeva C: Apoptosis in colorectal tumour cells: induction by the short chain fatty acids butyrate, propionate and acetate and by the bile salt deoxycholate. Int J Cancer 1995, 60(3):400-6.

25. Gibson PR, Rosella O, Wilson AJ, Mariadason JM, Rickard K, Byron K, et al: Colonic epithelial cell activation and the paradoxical effects of butyrate. Carcinogenesis 1999, 20(4):539-44

26. Daly K, Shirazi-Beechey SP: Microarray analysis of butyrate regulated genes in colonic epithelial cells. DNA Cell Biol 2006, 25(1):49-62.

27. Waby JS, Bingle CD, Corfe BM: Post-translational control of sp-family transcription factors. Curr Genomics 2008, 9(5):301-11.

28. Sgarra R, Rustighi A, Tessari MA, Di Bernardo J, Altamura S, Fusco A, et al: Nuclear phosphoproteins HMGA and their relationship with chromatin structure and cancer. FEBS Lett 2004, 574(1-3):1-8.

29. Luo J, Su F, Chen D, Shiloh A, Gu W: Deacetylation of p53 modulates its effect on cell growth and apoptosis. Nature 2000, 408(6810):377-81.

30. Corfe BM, Williams EA, Bury JP, Riley SA, Croucher LJ, Lai DY, et al: A study protocol to investigate the relationship between dietary fibre intake and fermentation, colon cell turnover, global protein acetylation and early carcinogenesis: the FACT study. BMC Cancer 2009, 9:332.

31. Seal CJ, Mathers JC: Comparative gastrointestinal and plasma cholesterol responses of rats fed on cholesterol-free diets supplemented with guar gum and sodium alginate. Br J Nutr 2001, 85(3):317-24.
32. Smedts F, Ramaekers F, Robben H, Pruszczynski M, van Muijen G, Lane B, et al: Changing patterns of keratin expression during progression of cervical intraepithelial neoplasia. Am J Pathol 1990, 136(3):657-68.

33. Herrmann $H$, Kreplak $L$, Aebi U: Isolation, characterization, and in vitro assembly of intermediate filaments. Methods Cell Biol 2004, 78:3-24.

34. Fujisaki J, Shimoda T: Expression of cytokeratin subtypes in colorectal mucosa, adenoma, and carcinoma. Gastroenterol Jpn 1993, 28(5):647-56.

35. Mizuuchi E, Semba S, Kodama Y, Yokozaki H: Down-modulation of keratin 8 phosphorylation levels by PRL-3 contributes to colorectal carcinoma progression. Int J Cancer 2009, 124(8):1802-10.

36. Doucet A, Butler GS, Rodriguez D, Prudova A, Overall CM: Metadegradomics: toward in vivo quantitative degradomics of proteolytic post-translational modifications of the cancer proteome. $\mathrm{Mol}$ Cell Proteomics 2008, 7(10):1925-51.

37. Duthie SJ: Folate and cancer: how DNA damage, repair and methylation impact on colon carcinogenesis. J Inherit Metab Dis 2010.

38. McOrist AL, Abell GC, Cooke C, Nyland K: Bacterial population dynamics and faecal short-chain fatty acid (SCFA) concentrations in healthy humans. Br J Nutr 2008, 100(1):138-46.

39. YU D, Bury J, Tiernan J, Waby JS, Staton C, Corfe B: Butyrate level and field cancerization have opposing effects on enteroendocrine cell number and neuropilin expression with early adenoma of the human colon. Molecular Cancer, accepted pending revision 2010.

\section{Pre-publication history}

The pre-publication history for this paper can be accessed here: http://www.biomedcentral.com/1471-230X/11/2/prepub

doi:10.1186/1471-230X-11-2

Cite this article as: Khan et al:: Keratin 8 expression in colon cancer associates with low faecal butyrate levels. BMC Gastroenterology 2011 $11: 2$

\section{Submit your next manuscript to BioMed Central and take full advantage of:}

- Convenient online submission

- Thorough peer review

- No space constraints or color figure charges

- Immediate publication on acceptance

- Inclusion in PubMed, CAS, Scopus and Google Scholar

- Research which is freely available for redistribution

Submit your manuscript at www.biomedcentral.com/submit
C) Biomed Central 